\title{
Efficacy of postoperative pain management in head and neck cancer patients
}

\author{
Ashley Hinther ${ }^{1}$, Steven C. Nakoneshny ${ }^{2}$, Shamir P. Chandarana ${ }^{1,2}$, T. Wayne Matthews ${ }^{1,2}$ and Joseph C. Dort ${ }^{1,2^{*}}$ (D)
}

\begin{abstract}
Background: Our study quantifies the effectiveness of perioperative pain control in a cohort of patients undergoing major head and neck surgery with free flap reconstruction. Our long-term goal is to improve pain control and thereby increase mobility, decrease postoperative complications and decrease hospital stay.

Methods: A retrospective analysis was performed at a tertiary, academic head and neck surgical oncology program in Calgary, Alberta, Canada from January 1, 2015 - December 31, 2015. Pain scores were recorded prospectively. Primary outcomes were frequency of postoperative pain assessments and pain intensity using the numeric rating scale.

Results: The cohort included 41 patients. Analysis was limited to pain scores recorded from postoperative days 1-14. There was an average of 7.3 pain measurements per day (SD 4.6, range 1-24) with the most frequent monitoring on postoperative days $1-4$.

Median pain scores ranged from 0 to 4.5 with the highest median score on postoperative day 6 . The daily maximum pain scores recorded ranged from 8 to 10 with scores of 10 recorded on postoperative days 1, 2, 3, 5, 7, 8, and 10 . Patients most frequently had inadequate pain control on postoperative days 1, 2, 4, and 5 with the majority occurring on postoperative day 1 .

Conclusions: Postoperative pain control could be improved at our centre. The frequency of pain assessments is also highly variable. Ongoing measurement, audit, and feedback of analgesic protocol effectiveness is an excellent first step in improving perioperative pain management in patients undergoing major head and neck cancer surgery with free flap reconstruction.
\end{abstract}

Keywords: Postoperative pain management, Postoperative pain control, ERAS, Enhanced recovery after surgery, Head and neck cancer, quality improvement

\section{Background}

Adequate pain control is a key element in successful recovery after major head and neck surgery. Inadequate postoperative pain management has been correlated with poor functional recovery [1]. Furthermore, continuous unrelieved post-operative pain can activate the pituitaryadrenal axis leading to immunosuppression resulting in postsurgical wound infection and poor wound healing [2-4]. Inadequate pain control can also reduce patient mobility, which can lead to deep vein thrombosis,

\footnotetext{
* Correspondence: jdort@ucalgary.ca

Presented at the 2017 AAO Meeting

'Department of Surgery, Section of Otolaryngology- Head and Neck Surgery, Cumming School of Medicine, University of Calgary, HRIC 2A02, 3280 Hospital Dr NW, Calgary, AB T2N 4Z6, Canada

${ }^{2}$ Ohlson Research Initiative, Arnie Charbonneau Cancer Institute, Cumming School of Medicine, University of Calgary, Calgary, AB, Canada
}

pulmonary embolism, and pneumonia [5, 6]. Effective postoperative pain control can shorten hospital stay, improve short-term post-operative outcomes, and decrease morbidity [7]. Additionally, poorly managed acute postoperative pain is often associated with chronic pain [8]. Major head and neck cancer resections with free flap reconstruction are lengthy and complex procedures and patients often require nasogastric and tracheotomy tubes. These interventions have a major impact on postoperative patient comfort and can make pain management challenging.

Adequate pain control implies consistent assessment of pain status and reliable delivery of appropriate analgesic medication. Important components of the pain assessment include determining the location of the pain as well as any aggravating or alleviating factors. SeIf-reported pain

(c) The Author(s). 2018 Open Access This article is distributed under the terms of the Creative Commons Attribution 4.0 International License (http://creativecommons.org/licenses/by/4.0/), which permits unrestricted use, distribution, and reproduction in any medium, provided you give appropriate credit to the original author(s) and the source, provide a link to the Creative Commons license, and indicate if changes were made. The Creative Commons Public Domain Dedication waiver (http://creativecommons.org/publicdomain/zero/1.0/) applies to the data made available in this article, unless otherwise stated. 
intensity is the most commonly assessed bedside pain dimension. Anderson et al. found that lack of pain assessment was a major barrier to achieving adequate pain control [9]. Optimal pain assessment requires standardization of schedule and format. Prior authors determined that greater than two pain assessments per day across 4 days is required to have an overall accurate assessment of patients' pain [10]. Ideally pain is reassessed after each intervention to not only determine the effectiveness of that intervention but also help determine what, if any, additional modifications are needed. Numerous pain intensity measures have been developed and validated. The numeric rating scale (NRS) uses a $0-10$ scale to rate the intensity of pain with 10 being the most intense pain [10-13]. As defined by the WHO, poorly controlled pain, or breakthrough pain, is defined as any score on the NRS greater than 3 [14-16].

Pain is prevalent in over $50 \%$ of cancer patients with the highest prevalence in patients with head and neck cancer (70\%) [17]. Orgill et al. reported that only $35 \%$ of postlaryngectomy patients received adequate and effective pain management [18]. Few studies have investigated the effectiveness of pain control in head and neck cancer patients. In most head and neck centers, narcotic analgesics form a major component of postoperative pain control regimens $[19,20]$. In our center, similar to others, most patients are managed with intravenous patient controlled analgesia (PCA) for the first five postoperative days and subsequently switched to a combination of narcotic and non-narcotic analgesics (acetaminophen and / or ibuprofen); however, narcotic analgesics have numerous adverse effects that include nausea and vomiting, constipation, sedation, and impaired mobilization [21, 22]. Furthermore, overuse of narcotics in the perioperative period can lead to subsequent drug dependence and its resulting personal and societal impacts.

The objective of this study was to better understand the effectiveness of our current approach to pain management in patients undergoing major head and neck surgery with free flap reconstruction. The type and effectiveness of our drug regimes, and the consistency and reliability of pain evaluation were of particular interest. We hypothesized there would be considerable variability in the evaluation and effectiveness of our approach to pain management. We also believed there would be generalizable findings that would inform our, and others', practice of pain management in this complex patient population. This information is a critical first step toward improving the overall management of pain in this high-risk patient population.

\section{Methods}

We performed a retrospective study of all patients undergoing head and neck cancer surgery with free flap reconstruction at the Foothills Medical Centre in Calgary, Alberta,
Canada from January 1, 2015 - December 31, 2015. Pain assessment scores were collected from an in-hospital electronic medical record for the duration of inpatient stay. Patient demographics and treatment data were collected from a prospectively annotated head and neck cancer database. Primary outcomes were frequency of postoperative pain assessments and pain intensity using the NRS. Secondary outcomes were time to mobilization and length of hospital stay.

Table 1 Patient demographics and clinical characteristics

\begin{tabular}{|c|c|}
\hline Characteristic & Number of subjects (\%) \\
\hline \multicolumn{2}{|l|}{ Gender } \\
\hline Male & $32(78 \%)$ \\
\hline Female & $9(22 \%)$ \\
\hline \multicolumn{2}{|l|}{ Age (yrs) } \\
\hline Mean (SD) & $61.2(12.3)$ \\
\hline Range & $23.6-82.0$ \\
\hline \multicolumn{2}{|l|}{ Primary site } \\
\hline Oral Cavity & $23(56 \%)$ \\
\hline Oropharynx & $3(7 \%)$ \\
\hline Larynx & $4(10 \%)$ \\
\hline Paranasal Sinus & $3(7 \%)$ \\
\hline Skin & $3(7 \%)$ \\
\hline Salivary Gland & $2(5 \%)$ \\
\hline Other Site & $3(7 \%)$ \\
\hline \multicolumn{2}{|l|}{ pT Classification } \\
\hline T0 & $3(7 \%)$ \\
\hline $\mathrm{T} 1$ & $6(15 \%)$ \\
\hline $\mathrm{T} 2$ & $13(32 \%)$ \\
\hline T3 & $3(7 \%)$ \\
\hline T4 & $11(27 \%)$ \\
\hline Tx & $5(12 \%)$ \\
\hline \multicolumn{2}{|l|}{ pN Classification } \\
\hline NO & $23(56 \%)$ \\
\hline $\mathrm{N} 1$ & $4(10 \%)$ \\
\hline N2 & 7 (17\%) \\
\hline Nx & $7(17 \%)$ \\
\hline \multicolumn{2}{|l|}{ Clinical Stage } \\
\hline 0 & $3(7 \%)$ \\
\hline । & $5(12 \%)$ \\
\hline$\|$ & $8(20 \%)$ \\
\hline III & $7(17 \%)$ \\
\hline IV & $13(32 \%)$ \\
\hline Not Stated & $5(12 \%)$ \\
\hline \multicolumn{2}{|l|}{ Length of Stay (d) } \\
\hline Mean (SD) & $11.6(5.5)$ \\
\hline Range & $4.0-29.0$ \\
\hline
\end{tabular}




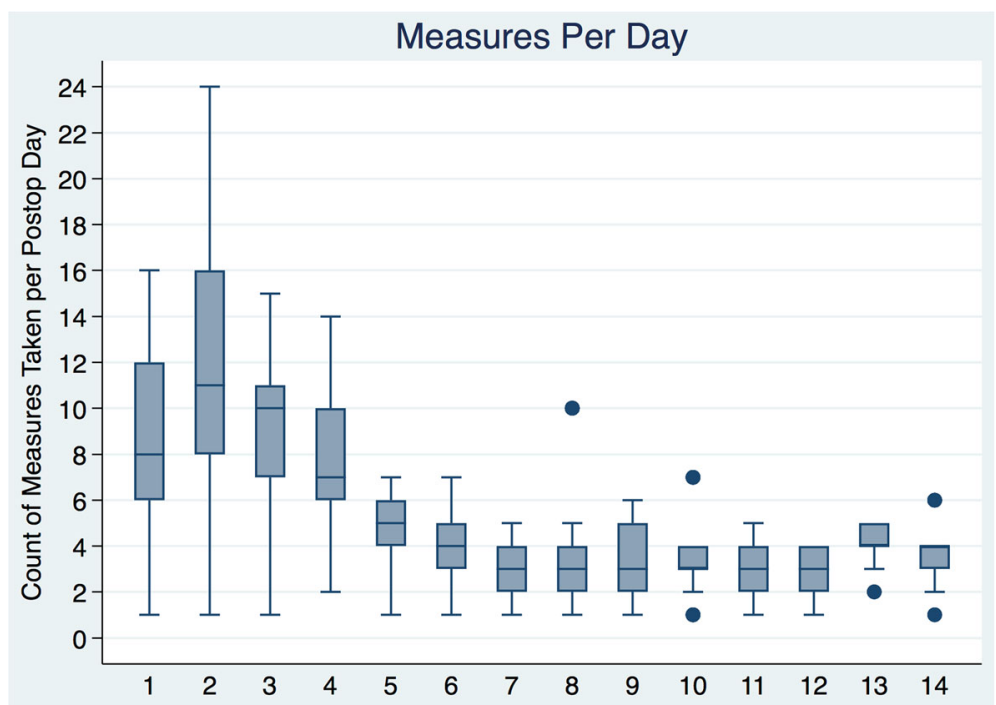

Fig. 1 Total number of pain assessments performed per postoperative day

Categorical variables are reported as proportions and continuous data are presented with means $+/$ - standard deviation as appropriate. All data were analyzed using Stata version 15 (Stata Corp, College Station, Tx, USA).

The authors used A pRoject Ethics Community Consensus Initiative (ARECCI) framework to assess for and mitigate ethical risks, including the ARECCI Ethics Screening Tool and the ARECCI Ethics Guidelines. The project was deemed a quality improvement initiative with a minimal risk $($ ARECCI score $=1)$.

\section{Results}

Clinical characteristics of the cohort $(n=41)$ are found in Table 1. The mean age was 61.2 years with a range of
23-82 years. Pain scores, using the NRS, were analyzed from postoperative days (POD) 1-14.

The mean length of hospital stay was 11.6 days with a range of 4-29 days. By POD 2, 71\% $(n=29)$ of patients were mobilized and $95 \%(n=39)$ were mobilized by POD 5 .

There was substantial variability in the number of daily pain assessments in the postoperative period (Fig. 1). On average, 7.3 pain measurements were performed daily (SD 4.6, range 1-24) with the most frequent monitoring taking place on PODs 1-4.

Figure 2 illustrates the proportion of patients receiving more than two pain assessments per day. Again, we found large variability in the number of pain assessments, with the greatest proportion of patients receiving more than

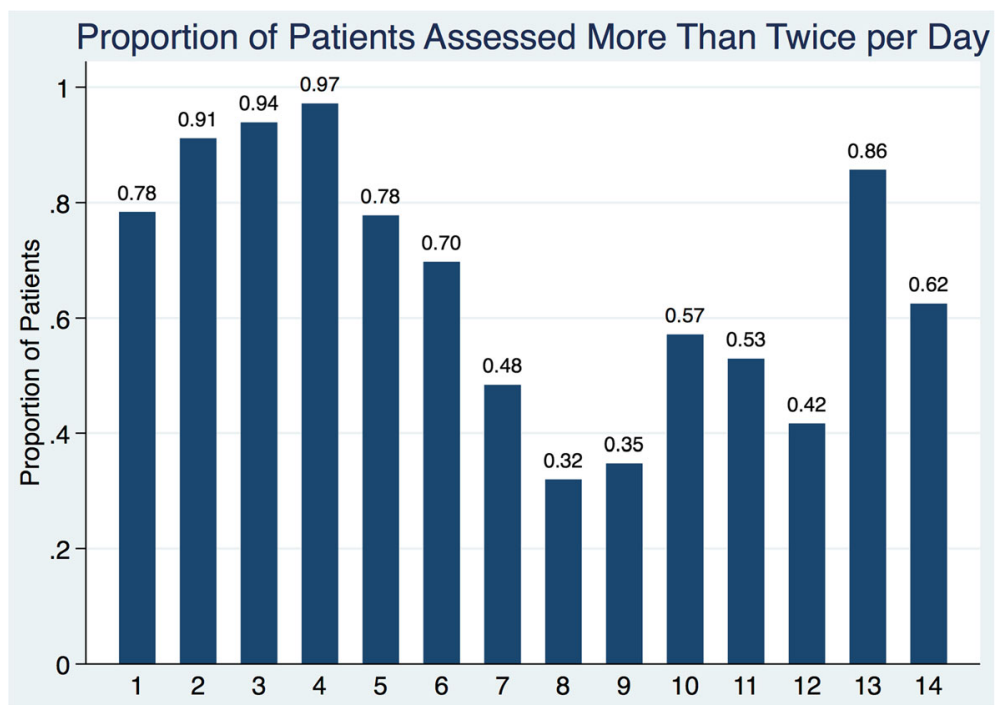

Fig. 2 Proportion of patients receiving $>2$ pain assessments per postoperative day 


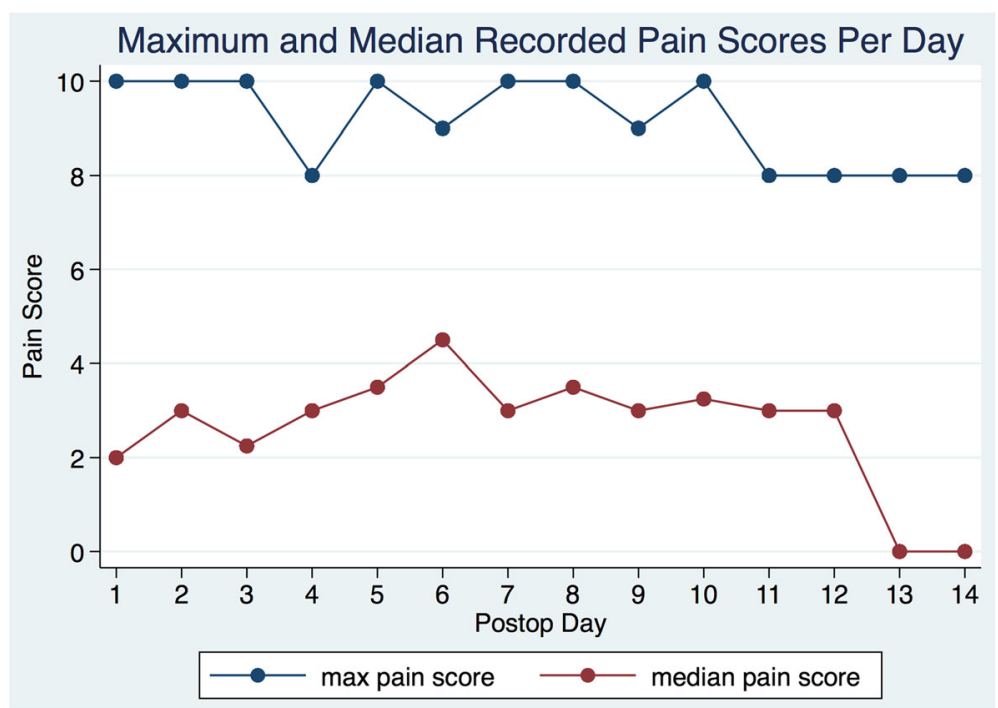

Fig. 3 Maximum and median pain scores per postoperative day

two assessments per day taking place on PODs 2, 3, 4, and 13. PODs 8 and 9 had the lowest proportion of patients receiving appropriate pain assessments with $32 \%$ and 35\% of patients receiving greater than two pain assessments that day, respectively. At no time did all of the patients receive an adequate number of daily pain assessments.

Figure 3 shows the maximum and median daily pain scores for all patients. Median pain scores ranged from 0 to 4.5 with the highest median score on POD 6 . The daily maximum pain scores recorded ranged from 8 to 10 with scores of 10 recorded on PODs 1, 2, 3, 5, 7, 8, and 10 .

Figure 4 demonstrates the efficacy of pain control by indicating the proportion of daily pain scores greater than 3 , reflecting poorly controlled pain. $31.5 \%$ (531/1684) of the total recorded pain scores were 5 or greater (not shown), signifying moderate to severe pain for at least part of the postoperative period. High scores were observed in 35 of 41 patients, indicating this is a common problem. Poor pain control was most frequent on PODs 1, 2, 4, 5, and 11 with the highest proportion occurring on POD 1. The highest proportion of patients with adequate pain control occurred on POD 8, 9, and 14.

\section{Discussion}

In this study, we found considerable variation in the frequency of pain assessments and the efficacy of pain control. Despite the frequent use of narcotic-based PCA regimes, many of our patients had pain scores

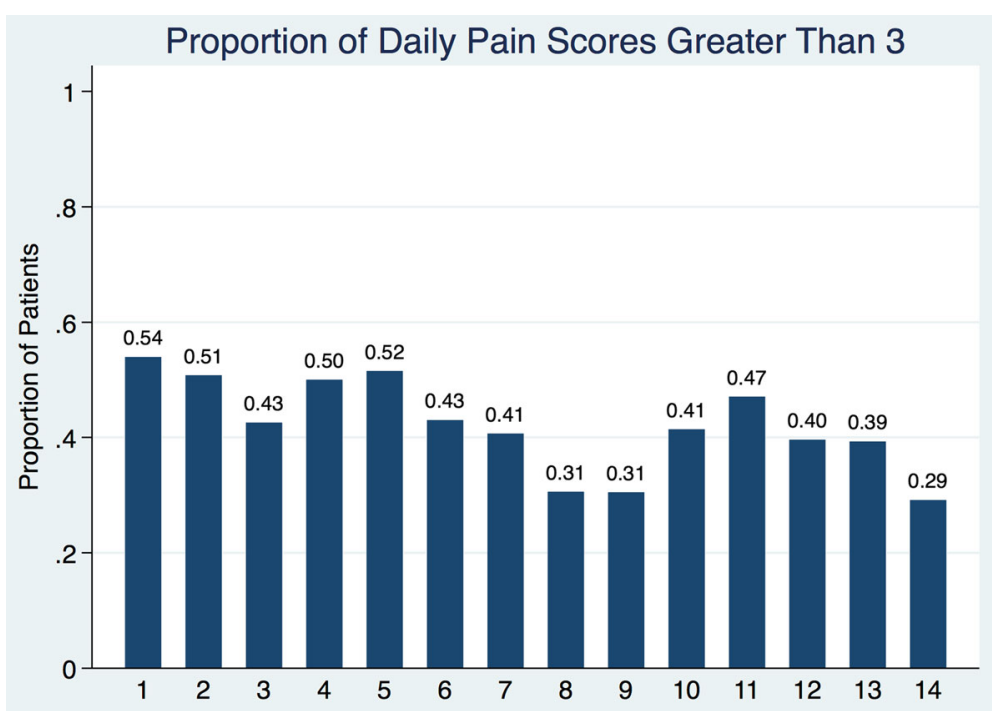

Fig. 4 Proportion of mean daily pain scores $>3$ 
greater than 3 with 35 of 41 patients having scores greater than or equal to 5 for at least some part of their hospital stay. These results indicate that current pain management is inconsistent and often ineffective. This study demonstrates there is an opportunity to standardize both postoperative pain assessments and pain management.

Inadequate pain control is a major barrier to a patient's postoperative recovery and can be a factor in the development of postoperative complications. Appropriate pain control not only takes into account the type of analgesic employed, but also the adequacy of pain assessment in order to ensure the patient's pain is controlled. Previous studies suggest that despite the existence of guidelines for managing oncologic pain, pain is inadequately treated in nearly half of cancer patients. Head and neck cancer patients have the highest pain prevalence at 70\% [14, 23]. These studies outline the importance of critically analyzing current pain management and addressing areas of weakness.

We are currently using the NRS for pain assessment. The NRS is a validated pain assessment tool that is easy to administer and record. Pain scores vary considerably throughout the day; therefore, the NRS must be administered frequently to adequately assess pain control. Jensen et al. demonstrated a minimum of three daily assessments per day should be performed for at least the first 4 postoperative days to provide a reliable pain assessment [24]. Although our results demonstrate a variable number of assessments throughout the day, we also determined there were no significant differences in average pain scores regardless if there were greater than two pain assessments per day (data not shown). We also found there is important inter-patient variability in the number of pain scores recorded per day and considerable intra-patient variability between the numbers of daily pain assessments. The WHO guidelines suggest poorly managed pain is any pain score on the NRS greater than 3 and scores of 5 or greater indicate moderate to severe pain. Figure 4 shows that a meaningful proportion of our patients are spending time in pain states of 3 or greater and our finding that $31.5 \%$ of total recorded pain scores were 5 or greater highlights that many patients (35 of 41) likely had less than optimal pain control.

Multimodal analgesic approaches used in other surgical populations minimize the use of narcotics and provide stable, reliable pain control, reduce postoperative nausea and vomiting, and improve ambulation for most patients [24-26]. The complex nature of head and neck cancer surgery suggests pain could be managed through a multimodal analgesic approach [20]. A 2014 randomized controlled study demonstrated decreased opioid requirements and length of hospital stay associated with pre-emptive intravenous paracetamol at the time of induction [27]. The French Oto-RhinoLaryngology- Head and Neck Surgery Society published guidelines pertaining to the management of postoperative pain in head and neck cancer patients. The French guidelines recommend multimodal analgesia; however, this recommendation is not evidence-based and relies upon professional consensus alone [23, 25, 28]. We therefore believe multimodal analgesia protocols represent an important avenue for further research in the head and neck patient population.

A major challenge in implementing multimodal analgesia is patients' medical comorbidities, which may contraindicate the use of multimodal protocols. Specifically, any patient with past history of peptic ulcer disease, renal failure, or liver disease will limit the use of NSAIDs and paracetamol.

This study is limited by its retrospective design that prevented an assessment of narcotic-induced complications and side effects. The retrospective design also meant we could not control the nature and frequency of analgesic administration. However, our primary goal was to assess adequacy of pain management and it was apparent that our current approach needs improvement.

This study is strengthened by its use of high quality administrative data that was collected prospectively at the point of care. Such data are highly reliable and we are confident in its accuracy and reliability. Few studies of perioperative pain management in major head and neck cancer surgery with free flap reconstruction have been published. Studies that have been published show a high reliance on narcotic based pain control $[8,9]$. We believe that multimodal analgesic protocols, as shown in other surgical disciplines, will reduce the need for postoperative narcotics in head and neck cancer patients and the complications that attend their use [16-18].

\section{Conclusions}

We conclude that in a tertiary academic head and neck surgical oncology program there is significant variation in the number of pain assessments and in the adequacy of pain control in patients undergoing major head and neck cancer surgery with free flap reconstruction. Our results suggest that ongoing measurement, audit, and feedback of analgesic protocol effectiveness is an excellent first step in improving perioperative pain management in patients undergoing major head and neck cancer surgery with free flap reconstruction.

\section{Abbreviations}

ARECCl: A pRoject Ethics Community Consensus Initiative; NRS: Numeric rating scale; PCA: Patient-controlled analgesia; POD: Postoperative day; WHO: World Health Organization 


\section{Acknowledgements}

We gratefully acknowledge the Ohlson Research Initiative for supporting this project.

\section{Funding}

Funding support for this research was provided by a grant from the Ohlson Research Initiative. The funder had no role in study design, or collection, analysis, interpretation of data, or in the writing of the manuscript.

\section{Availability of data and materials}

The datasets generated and/ analyzed during this study available from the corresponding author on reasonable request.

\section{Authors' contributions}

$\mathrm{AH}$ participated in data analysis, interpretation of the data, and participated in writing and preparation of the manuscript. JCD developed the study concept and design, acquired the data, was involved in the analysis and interpretation, and co-wrote and critically revised the manuscript. SCN assisted with study design, performed data analysis and interpretation of the data, and helped draft and critically revise the manuscript. SPC and TWM assisted in the interpretation of the data and critically revised the manuscript. All authors give final approval for the manuscript to be published and agree to be accountable for it.

\section{Ethics approval and consent to participate}

The authors used A pRoject Ethics Community Consensus Initiative (ARECC) framework to assess for and mitigate ethical risks, including the ARECCI Ethics Screening Tool and the ARECCI Ethics Guidelines. The project was deemed a quality improvement initiative with a minimal risk (ARECCI score $=1$ ).

\section{Competing interests}

The authors declare that they have no competing interests.

\section{Publisher's Note}

Springer Nature remains neutral with regard to jurisdictional claims in published maps and institutional affiliations.

Received: 20 November 2017 Accepted: 17 April 2018

Published online: 02 May 2018

\section{References}

1. Capdevila X, Barthelet $Y$, Biboulet P, Ryckwaert $Y$, Rubenovitch J, d'Athis $F$. Effects of perioperative analgesic technique on the surgical outcome and duration of rehabilitation after major knee surgery. Anesthesiology. 1999;91(1):8-15

2. Wells N, Paeroa C, McCaffery M. Chapter 17 improving the quality of care through pain assessment and management. In: Hughes RG, ed. Patient safety and quality: an evidence-based handbook for nurses: Rockville (MD): Agency for Healthcare Research and Quality (US); 2008.

3. Barr J, Boulind C, Foster JD, et al. Impact of analgesic modality on stress response following laparoscopic colorectal surgery: a post-hoc analysis of a randomised controlled trial. Tech Coloproctol. 2015;19(4):231-9.

4. Scott MJ, Baldini G, Fearon KC, et al. Enhanced recovery after surgery (ERAS) for gastrointestinal surgery, part 1: pathophysiological considerations. Acta Anaesthesiol Scand. 2015;59(10):1212-31.

5. Yeung JK, Dautremont JF, Harrop AR, et al. Reduction of pulmonary complications and hospital length of stay with a clinical care pathway after head and neck reconstruction. Plast Reconstr Surg. 2014;133(6):1477-84.

6. Yeung JK, Harrop R, McCreary O, et al. Delayed mobilization after microsurgical reconstruction: an independent risk factor for pneumonia. Laryngoscope. 2013;123(12):2996-3000.

7. Wick EC, Grant MC, Wu CL. Postoperative multimodal analgesia pain management with nonopioid analgesics and techniques: a review. JAMA Surg. 2017:152(7):691-7.

8. Kuo P-Y, Williams JE. Pain control in head and neck Cancer. In: Agulnik M, editor. Head and neck Cancer: InTech; 2012. p. 351-70.

9. Anderson $\mathrm{KO}$, Mendoza TR, Valero $\mathrm{V}$, et al. Minority cancer patients and their providers: pain management attitudes and practice. Cancer. 2000; 88(8):1929-38.

10. Jensen MP, McFarland CA. Increasing the reliability and validity of pain intensity measurement in chronic pain patients. Pain. 1993;55(2):195-203.
11. Hawker GA, Mian S, Kendzerska T, French M. Measures of adult pain: visual analog scale for pain (VAS pain), numeric rating scale for pain (NRS pain), McGill pain questionnaire (MPQ), short-form McGill pain questionnaire (SF$M P Q$ ), chronic pain grade scale (CPGS), short Form-36 bodily pain scale (SF36 BPS), and measure of intermittent and constant osteoarthritis pain (ICOAP). Arthritis Care Res (Hoboken). 2011;63(Suppl 11):S240-52.

12. Jensen MP, Karoly P, O'Riordan EF, Bland F Jr, Burns RS. The subjective experience of acute pain. An assessment of the utility of 10 indices. Clin J Pain. 1989:5(2):153-9.

13. Williamson A, Hoggart B. Pain: a review of three commonly used pain rating scales. J Clin Nurs. 2005:14(7):798-804.

14. Grond S, Zech D, Lynch J, Diefenbach C, Schug SA, Lehmann KA. Validation of World Health Organization guidelines for pain relief in head and neck cancer. A prospective study. Ann Otol Rhinol Laryngol. 1993;102(5):342-8.

15. Zech DF, Grond S, Lynch J, Hertel D, Lehmann KA. Validation of World Health Organization guidelines for cancer pain relief: a 10-year prospective study. Pain. 1995;63(1):65-76.

16. Organization WH. Cancer pain relief. 1996. http://apps.who.int/iris/bitstream/ 10665/37896/1/9241544821.pdf. Accessed May 2017.

17. van den Beuken-van Everdingen MH, de Rijke JM, Kessels AG, Schouten HC, van Kleef M, Patijn J. Prevalence of pain in patients with cancer: a systematic review of the past 40 years. Ann Oncol. 2007:18(9):1437-49.

18. Orgill R, Krempl GA, Medina JE. Acute pain management following laryngectomy. Arch Otolaryngol Head Neck Surg. 2002:128(7):829-32.

19. Bianchini C, Malago M, Crema L, et al. Post-operative pain management in head and neck cancer patients: predictive factors and efficacy of therapy. Acta Otorhinolaryngol Ital. 2016;36(2):91-6.

20. Bianchini C, Maldotti F, Crema L, Malago M, Ciorba A. Pain in head and neck cancer: prevalence and possible predictive factors. J BUON. 2014;19(3):592-7.

21. Tan M, Law LS, Gan TJ. Optimizing pain management to facilitate enhanced recovery after surgery pathways. Can J Anaesth. 2015;62(2):203-18.

22. Oderda GM, Evans RS, Lloyd J, et al. Cost of opioid-related adverse drug events in surgical patients. J Pain Symptom Manag. 2003:25(3):276-83.

23. Binczak M, Navez $M$, Perrichon C, et al. Management of somatic pain induced by head-and-neck cancer treatment: definition and assessment. Guidelines of the French Oto-rhino-laryngology- head and neck surgery society (SFORL). Eur Ann Otorhinolaryngol Head Neck Dis. 2014;131(4):243-7.

24. Khan SK, Malviya A, Muller SD, et al. Reduced short-term complications and mortality following enhanced recovery primary hip and knee arthroplasty: results from 6,000 consecutive procedures. Acta Orthop. 2014;85(1):26-31.

25. Kalogera E, Bakkum-Gamez JN, Jankowski CJ, et al. Enhanced recovery in gynecologic surgery. Obstet Gynecol. 2013;122(2 Pt 1):319-28.

26. Miller TE, Thacker JK, White WD, et al. Reduced length of hospital stay in colorectal surgery after implementation of an enhanced recovery protocol. Anesth Analg. 2014;118(5):1052-61.

27. Majumdar S, Das A, Kundu R, Mukherjee D, Hazra B, Mitra T. Intravenous paracetamol infusion: superior pain management and earlier discharge from hospital in patients undergoing palliative head-neck cancer surgery. Perspect Clin Res. 2014;5(4):172-7.

28. Espitalier F, Testelin S, Blanchard D, et al. Management of somatic pain induced by treatment of head and neck cancer: postoperative pain. Guidelines of the French Oto-rhino-laryngology-head and neck surgery society (SFORL). Eur Ann Otorhinolaryngol Head Neck Dis. 2014;131(4):249-52.
- fast, convenient online submission

- thorough peer review by experienced researchers in your field

- rapid publication on acceptance

- support for research data, including large and complex data types

- gold Open Access which fosters wider collaboration and increased citations

- maximum visibility for your research: over $100 \mathrm{M}$ website views per year

\section{At BMC, research is always in progress.}

Learn more biomedcentral.com/submissions 